\title{
Morphology-controlled synthesis of hexagonal boron nitride crystals by chemical vapor deposition
}

\begin{abstract}
Synthesis of hexagonal boron nitride $(\mathrm{hBN})$ crystals with a controlled morphology is a major challenge due to various kinetic and thermodynamic factors at the edge. Supplying BN building blocks by heating precursor ammonia borane at a temperature of $90{ }^{\circ} \mathrm{C}$ produced triangular crystals, whereas, at higher heating $\left(130{ }^{\circ} \mathrm{C}\right), \mathrm{hBN}$ crystals with a hexagonal morphology were observed. The shape of crystals could also be modulated from hexagonal to triangular under a continuous reduced supply of BN building blocks. We attributed these phenomena to a different growth mechanism dependent on the concentration of BN radicals in the growth region. With a low supply of BN building blocks, crystal growth is the edge attachment limited, producing triangles, whereas, under higher concentration of BN building blocks, crystal growth is limited by diffusion producing hexagons. The presence of alternating $\mathrm{B}$ and $\mathrm{N}$ terminated vertices in hexagonal $\mathrm{hBN}$ crystals offers the possibility of $\mathrm{hBN}$ /graphene in-plane heterostructure synthesis with $\mathrm{B}-\mathrm{C}$ and $\mathrm{N}-\mathrm{C}$ bonding at the interface, which is not possible with $\mathrm{N}$ terminated triangular crystals. Supplying the controlled amount of $\mathrm{BN}$ building blocks to synthesize triangular and hexagonal crystals and modulation from hexagons to triangles is a major achievement (in the present work) due to single parameter control over the experiment.
\end{abstract}

Keyword: Morphology-controlled synthesis; Hexagonal boron nitride crystals; Chemical vapor deposition 